\title{
Bienestar emocional, psicológico y social y variables demográficas en población adulta de la ciudad de Buenos Aires
}

\author{
Vanesa Góngora ${ }^{1}$ y Alejandro Castro Solano ${ }^{2}$
}

\begin{abstract}
RESUMEN
El estudio tuvo como objetivo 1) comparar los niveles de satisfacción con la vida, el significado en la vida y la satisfacción en distintas áreas vitales, depresión y síntomas somáticos entre adultos de la ciudad de Buenos Aires en las distintas categorias del continuo de salud mental y 2) comparar los niveles de bienestar emocional, psicológico y social en adultos de la ciudad de Buenos Aires según sexo, grupo de edad, ocupación y nivel educativo. Se utilizó una muestra de 460 adultos (18 a 85 años) de la ciudad de Buenos Aires. Se administraron el Mental Health Continuum-Short Form, la escala de depresión CES-D, la escala de síntomas somáticos de Schaffer, la escala de Satisfacción con la vida, el cuestionario de Significado en la Vida y el Personal Well-being Index. Los resultados indicaron que aquellos adultos que se encontraban en la categoría de salud mental floreciente presentaban mayores niveles de satisfacción con la vida, satisfacción en distintas áreas vitales y presencia de significado en la vida así como menor depresión y síntomas somáticos. Los adultos con mayor nivel educativo mostraron mayores niveles de bienestar psicológico y social. Se encontró un mayor nivel de bienestar social en adultos de edad media (30-60 años) y en trabajadores independientes.
\end{abstract}

Palabras clave: salud mental, bienestar, adultos, psicología positiva.

\section{Emotional, Psychological and Social Well-Being, and Demographic Variables in the Adult Population of Buenos Aires City}

\begin{abstract}
The study aimed to 1) compare the levels of satisfaction with life, meaning in life, satisfaction in different areas of life, depression and somatic symptoms in adults from the city of Buenos Aires among the mental health continuum categories and 2) to compare the levels of emotional, psychological and social well-being in adults from Buenos Aires by sex, age group, occupation and educational level. A sample of 460 adults (18-85 years old) from the city of Buenos Aires was used. The Mental Health Continuum-Short Form, CES-D Depression Scale, Schaffer Somatic Symptoms Scale, the Satisfaction with Life Scale, the Meaning in Life Questionnaire and Personal Well-being Index were used. The results indicated that adults who were in the flourishing mental health category had higher levels of satisfaction with life, satisfaction in different areas of life, and presence of meaning in life as well as lower level of depression and somatic symptoms. Adults with higher levels of education showed higher levels of psychological and social well-being. Higher social well-being was found in middle-aged adults (30-60 years) and in independent workers.
\end{abstract}

Keywords: mental health, well-being, adults, positive psychology.

1 y 2 Consejo Nacional de Investigaciones Científicas y Técnicas (CONICET), Universidad de Palermo, Argentina; gongoravanesa@gmail.com 
La evaluación y el desarrollo del bienestar en las personas es un tema de creciente interés dentro de la psicologia. Tradicionalmente el bienestar ha sido conceptualizado desde dos perspectivas diferentes (Ryan \& Deci, 2001). La primera está en relación con la tradición hedónica e incluye el estudio de la satisfacción con la vida, del bienestar subjetivo y de las emociones positivas (Diener, 2009; Lyubomirsky \& Lepper, 1999; Pavot \& Diener, 2008). Desde esta línea una persona feliz es aquella que experimentó mayor frecuencia de emociones positivas que negativas (Henderson \& Knight, 2012). La segunda perspectiva considera que el bienestar es eudaemónico. En esta orientación el bienestar se alcanza cuando se logran complejas metas vitales con un alto grado de significado personal (Henderson \& Knight, 2012). A pesar de que muchos estudios han considerado el bienestar desde un abordaje unilateral, o bien hedónico o eudaemónico, la investigación reciente reconoce que ambas perspectivas son distintas (Diener et al., 2009) y en cierto sentido complementarias (Ryan \& Deci, 2001). Las teorías más actuales del bienestar integran la perspectiva hedónica y la eudaemónica y denominan flourishing o florecimiento al estado en el que ambos tipos de bienestar se encuentran en altos niveles (Hone, Jarden, Schofield, \& Duncan, 2014); en otras palabras, el bienestar implica no solo sentirse bien (hedónico) sino también funcionar bien (eudaemónico). Diferentes autores han desarrollado la conceptualización y operacionalización del flourishing: Keyes (2002), Diener et al. (2010), Seligman (2011) y Huppert y So (2013). Dentro de estas formulaciones, la de Keyes (2002, 2005) es la que más desarrollo e investigaciones ha tenido.

Keyes define al flourishing como altos niveles de: a) bienestar emocional, entendido en términos de afectos positivos y satisfacción con la vida (bienestar hedónico); b) bienestar psicológico en términos de la teoría del bienestar psicológico de Ryff (Ryff, 1989) (autonomía, control, crecimiento personal, relaciones personales, autoaceptación y propósito) y c) bienestar social (incluye las facetas de aceptación, actualización, contribución social, coherencia e integración social) (Keyes, 2005). Para este autor, el flourishing o florecimiento sería el estado óptimo de la salud mental, en tanto el languishing o languidecimiento sería un bajo nivel de salud mental (bajo bienestar hedónico y eudaemónico). Una categoría intermedia la constituiría la salud mental moderada, formándose así un continuo de salud mental: languishingsalud mental moderada-flourishing (Keyes \& López, 2002). Debe señalarse que Keyes (2005) utiliza el término salud mental (y no bienestar) para referirse a la clasificación que hace del bienestar emocional, psicológico y social en forma conjunta.

Los diferentes estudios internacionales han demostrado amplias variaciones en las tasas de prevalencia del flourishing en adultos de distintos países, que se sitúa entre un $8 \%$ en Corea del Sur (Lim, Ko, Shin, \& Cho, 2013) al $72.5 \%$ en Canadá (Gilmour, 2014). En casi todos los países estudiados, las categorias de la salud mental moderada y flourishing representan a la mayoría de la población adulta; el languidecimiento es una categoría de menor representación cuyas tasas oscilan entre un $1.5 \%$ en Canadá a un 21.2\% en Corea del Sur (Gilmour, 2014; Lim et al., 2013).

La investigación ha apoyado la hipótesis de que los adultos con alta salud mental (flourishing) funcionaban en todos los aspectos mejor que los adultos con salud mental moderada, que a su vez funcionaban mejor que los adultos en la categoría languishing. Una salud mental floreciente se asoció con menores limitaciones en las actividades diarias, menor número de 
enfermedades, condiciones físicas crónicas (Keyes, 2005), disminución del riesgo de diversas causas de mortalidad (Keyes \& Simoes, 2012) y menores sintomas somáticos (Karaś, Cieciuch, \& Keyes, 2014; Petrillo, Capone, Caso, \& Keyes, 2015). Por otro lado, también se relacionó con altos niveles de funcionamiento psicosocial, en cuanto a mayor claridad en los objetivos vitales, mejor autoestima, mayor autoeficacia, mayor presencia de significado en la vida y mayor percepción de vínculos de intimidad (por ejemplo, con la familia y amigos) (Karaś et al., 2014; Keyes, 2007; Petrillo et al., 2015). En lo que respecta a la salud mental, altos niveles de flourishing se asociaron con menor aparición de enfermedades mentales en adultos, menores niveles de depresión, ansiedad generalizada, riesgo suicida, abuso de sustancias, dependencia de alcohol y trastorno de pánico en adultos (Gilmour, 2014; Karaś et al., 2014; Keyes, 2005; Keyes, Dhingra, \& Simoes, 2010; Keyes et al., 2011; Petrillo et al., 2015; Yin, He, \& Fu, 2013).

Keyes (2002) sostuvo que las variables demográficas tales como el sexo, edad, nivel educativo u ocupacional podrian afectar el continuo de salud mental. Sin embargo, los estudios internacionales han encontrado resultados dispares. Con respecto al sexo, los estudios encontraron mayores niveles de salud mental en hombres italianos y estadounidenses (Keyes, 2005; Petrillo et al., 2015), sin embargo Westerof y Keyes (2010) hallaron mayores niveles en mujeres holandesas; otras investigaciones no encontraron diferencias entre hombres y mujeres canadienses, coreanos y chinos (Gilmour, 2014; Lim et al., 2013; Yin et al., 2013). En cuanto a los grupos de edad, los resultados son aún más disímiles. Mayores niveles de salud mental se encontraron en adultos jóvenes coreanos y polacos, adultos mayores estadounidenses y canadienses, y adultos de edad media (30-60 años) italianos, en tanto no se encontraron diferencias en adultos chinos y holandeses (Gilmour, 2014; Karaś et al., 2014; Keyes, 2005; Lim et al., 2013; Petrillo et al., 2015; Westerhof \& Keyes, 2010; Yin et al., 2013). Por otro lado, un mayor nivel educativo se asoció con mayores niveles de salud mental en adultos chinos, polacos, estadounidenses y coreanos (Karaś et al., 2014; Keyes, 2005; Lim et al., 2013; Yin et al., 2013). No se hallaron diferencias significativas en los niveles de salud mental por tipo de empleo en adultos estadounidenses y canadienses, sin embargo, los trabajadores independientes chinos presentaron mayor salud mental. El hecho de estar desempleado se vinculó con menores niveles de salud mental en adultos estadounidenses y canadienses. En consonancia con esto hallazgos, las amas de casa coreanas mostraron menor nivel de salud mental (Gilmour, 2014; Keyes, 2005; Lim et al., 2013).

Hasta el momento las investigaciones realizadas en Argentina se han centrado en la validación del MHC-SF (Mental Health Continuum-Short Form), instrumento para evaluar la salud mental, y en la prevalencia del continuo de salud mental en población adulta de la ciudad de Buenos Aires. Los estudios confirmaron el ajuste de los datos locales a un modelo de tres factores y esta estructura se mostró invariante por sexo y edad (Lupano Perugini, de la Iglesia, Castro Solano, \& Keyes, 2017). En cuanto a la prevalencia, la mayoria de los adultos de la ciudad de Buenos Aires presentaron una salud mental moderada $(54.9 \%)$ o floreciente $(39.7 \%)$ y solo una minoria $(5.7 \%)$ evidenciaba una salud mental languideciente. El hecho de tener un trastorno mental disminuía significativamente el estado de salud mental y las personas tenían una salud mental más languideciente y menos floreciente (Góngora \& Castro Solano, 2017). Sin embargo, los estudios son escasos y se hace necesario 
extender las investigaciones sobre la base de este modelo en población argentina.

En vista de la disparidad en los resultados previos sobre el bienestar y las variables demográficas así como la necesidad de investigar la relación entre las categorias de salud mental y otras variables positivas y psicopatológicas en población argentina se plantea la presente investigación.

Los objetivos de este estudio son: 1) comparar los niveles de satisfacción con la vida, el significado en la vida y la satisfacción en distintas áreas vitales, depresión y síntomas somáticos entre adultos de la ciudad de Buenos Aires en las distintas categorias del continuo de salud mental y 2) comparar los niveles de bienestar emocional, psicológico y social en adultos de la ciudad de Buenos Aires según sexo, grupo de edad, ocupación y nivel educativo.

Teniendo en cuenta los estudios previos internacionales, se espera encontrar mayores niveles de satisfacción con la vida, de significado en la vida, mayor satisfacción en distintas áreas vitales así como menores niveles de depresión y síntomas somáticos en adultos de la ciudad de Buenos Aires clasificados en la categoría de flourishing. Por otro lado, se espera que haya mayores niveles de bienestar en adultos con mayor nivel educativo.

\section{METODOLOGÍA}

\section{Muestra}

La muestra de tipo no probabilística, intencional, estuvo compuesta por 460 personas adultas, la mitad fueron hombres $(n=230,50 \%)$ y la otra mitad, mujeres $(n=230,50 \%)$. La división de la muestra en mitades por sexo se debió a la búsqueda de igual representación de hombres y mujeres en los resultados. La edad media fue de 40.5 años $(D E=14.1)$, que va desde 18 a 85 años. La mayoría de los participantes nacieron en la región metropolitana de Buenos Aires $(n=292,63.4 \%), 22.6 \%(n=104)$ en otras ciudades de Argentina, $11.1 \%(n=51)$ eran extranjeros y el $2.9 \%(n=13)$ no dio ninguna información. En relación con el nivel más alto de educación alcanzado, el 8.8 $\%(n=41)$ de la muestra tenía educación básica, el 48,3 \% $(n=222)$ educación media, el 37,5 \% ( $n=172)$ educación terciaria o universitaria y solo el 5,3\% ( $n$ $=25$ ) recibió formación de postgrado. Con respecto al nivel de ocupación, 8.7 $\%(n=40)$ eran empleadores, $56.7 \%(n=261)$ empleados, el $16.3 \%(n=75)$ trabajadores independientes, el $5.2 \%(n=24)$ estaba jubilado o pensionado, el $1.3 \%(n=6)$ estaba desempleado y buscaba trabajo, el $3.7 \%(n=17)$ era ama de casa o trabajador sin salario y un $8 \%(n=37)$ no trabajaba y no buscaba trabajo.

\section{Instrumentos}

Mental Health Continuum- Short Form (MHC-SF). Este instrumento de 14 items (Keyes, 2005) evalúa el grado de: a) bienestar emocional (afectos positivos y satisfacción con la vida); b) bienestar social (incluye las facetas de aceptación, actualización, contribución social, coherencia e integración social); c) bienestar psicológico (autonomía, control, crecimiento personal, relaciones personales, autoaceptación y propósito) (Ryff, 1989). El MHC-SF ha mostrado buenas evidencias de consistencia interna $(\alpha>$.70) y validez discriminante en muestras de adultos de diversos países. La estructura de tres factores de la escala (emocional, psicológica y social) ha sido confirmada en los diversos 
estudios (Gallagher, López, \& Preacher, 2009; Joshanloo, Wissing, Khumalo, \& Lamers, 2013). Los estudios de validación de este instrumento en Argentina han confirmado la estructura factorial del instrumento y han dado evidencia de una buena validez convergente y consistencia interna en población adulta (Lupano Perugini et al., 2017). En esta muestra, las consistencias internas fueron $\alpha=.87$ para bienestar emocional, $\alpha=.80$ para bienestar psicológico y $\alpha=.76$ para bienestar social.

Escala de depresión CES-D. Es un instrumento de screening especialmente diseñado para la detección de patologías depresivas en población adulta general no consultante (Radloff, 1977). Se utiliza para realizar estudios epidemiológicos. Posee 20 items, de los cuales 4 son inversos, que permiten obtener una puntuación total. Los análisis estadísticos verifican que posee alta consistencia interna así como confiabilidad test-retest, y presenta alta correlación con otras técnicas estandarizadas que evalúan sintomatología depresiva (Casullo, 1998). En esta muestra la consistencia interna para la escala fue $\alpha=.88$.

Escala de sintomas somáticos de Schaffer (Somatic Symptoms Index). Este cuestionario evalúa la severidad de síntomas somáticos a través de 10 ítems (Schafer \& Ferraro, 2013). Los ítems se refieren a la experimentación de distintos síntomas somáticos en el trascurso de los últimos 30 días con opciones de respuesta que van desde nada (1) a casi todos los días (6). Todos los ítems tienen puntaje directo ( 1 a 6 ), a mayor puntuación, mayor severidad de sintomas. La consistencia interna de la escala en esta muestra es $\alpha=.82$.

Escala de Satisfacción con la vida (SWLS). Es una escala de cinco ítems con formato de respuesta likert en 7 categorias y examina el grado de satisfacción global con la vida (Diener, Emmons, Larsen, \& Griffin, 1985). La escala es utilizada internacionalmente para la evaluación del bienestar en tanto componente cognitivo de la satisfacción. Diferentes estudios empíricos han demostrado su validez y fiabilidad (Diener et al., 1985; Pavot, Diener, Colvin, \& Sandvik, 1991). La escala ha sido validada en población argentina (Castro Solano \& Díaz Morales, 2000). En esta muestra el nivel de consistencia interna es $\alpha=.88$.

Cuestionario de Significado en la Vida (MLQ). Este instrumento consta de 10 ítems que evalúan el sentido de la vida (Steger, Frazier, Oishi, \& Kaler, 2006). El MLQ está conformado por dos subescalas con 5 ítems cada una: Presencia y Búsqueda de sentido obteniéndose puntuaciones independientes para cada dimensión. El cuestionario presenta opciones de respuesta tipo Likert que van de absolutamente falso, que se puntúa 1, a absolutamente verdadero, que se puntúa 7 . Los estudios de validación local replicaron la estructura de dos factores a través del análisis factorial exploratorio $\mathrm{y}$ confirmatorio (Góngora \& Castro Solano, 2011). En la presente muestra el nivel de consistencia interna es $\alpha=.87$ para Presencia $y \alpha=.89$ para Búsqueda.

Personal Well-being Index (PWI; Cummins, 1998). Evalúa la satisfacción en diferentes dominios vitales (e.g., nivel de vida, salud, logros, relaciones personales, seguridad, comunidad, seguridad futura, espiritualidad). El PWI utiliza una escala del 0 (cero) al 10 (diez), en la cual el 0 corresponde a no estar satisfecho y el 10 corresponde al más alto nivel de satisfacción. E1 instrumento ha sido adaptado y utilizado en 51 países, incluida la Argentina (Casas et al., 2012; Tonón, 2012). La consistencia interna para esta muestra es $\alpha=.88$. 


\section{Procedimiento}

Se solicitó a los participantes la firma de un consentimiento informado conforme a las normas éticas que regulan la evaluación psicológica de personas. Las administraciones se realizaron en forma individual en una sesión de aproximadamente 45 minutos. Pasantes de investigación de la carrera de Psicología de la Universidad de Palermo, previamente entrenados, colaboraron en las tareas de administración, bajo la supervisión de los autores de este trabajo. El análisis de los datos se realizó con el programa estadístico SPSS 18.

\section{Análisis de datos}

Para clasificar los niveles de salud mental según el MHC-SF se utilizó el procedimiento estándar diseñado por Keyes (2005). Las personas que reportaron experimentar "todos los días" o "casi todos los dias" al menos uno de los tres indicadores de bienestar hedónico y al menos seis de los once indicadores de funcionamiento positivo durante el pasado mes se ubicaron en la categoría de salud mental floreciente. Las personas que reportaron niveles bajos (es decir, "nunca" o "una o dos veces" durante el mes pasado) en al menos un indicador de bienestar hedónico y bajos niveles en al menos seis indicadores de funcionamiento positivo fueron diagnosticados con salud mental languideciente. El resto de los participantes que no entraban en las dos categorias anteriores se clasificaron con salud mental moderada.

Se efectuó en primer lugar un cálculo de frecuencia de los niveles de salud mental. Posteriormente, se realizaron pruebas de análisis de varianza (ANOVA) para comparar las variables: depresión (CES-D), significado en la vida (MLQ), satisfacción con la vida (SWLS), satisfacción con distintas áreas vitales (PWI) y sintomas somáticos (Shaffer) de acuerdo a las categorias de salud mental evaluadas por el MHC-SF. Para calcular diferencias en los niveles de bienestar de acuerdo a variables demográficas se utilizaron las tres subescalas del MHC-SF: bienestar emocional, bienestar social y bienestar psicológico como variables dependientes y las variables demográficas como factores. En el caso de diferencias por sexo, se efectuó una prueba t para muestras independientes, en tanto que para nivel de educación, grupos de edad, y ocupación se calcularon análisis univariados de varianza (ANOVA).

\section{RESULTADOS}

\section{Comparación de variables de estudio de acuerdo a los niveles de salud mental}

Se calcularon los niveles de salud mental utilizando el MHC-SF en la muestra de estudio. Una gran parte de la muestra mostró una salud mental moderada (59.3\%) y un porcentaje significativo, una salud mental floreciente $(35.2 \%)$, en tanto que solo un $5.5 \%$ fue clasificado como salud mental languideciente.

Se realizó un análisis univariado de Varianza con análisis post-hoc de Games-Howell (tabla 1). Se seleccionó este procedimiento post-hoc debido a que el análisis de homogeneidad de varianzas de Levene demostró que estas eran desiguales entre los grupos. 
Los resultados del análisis mostraron que los individuos con salud mental floreciente presentaban menores niveles de depresión y de sintomas somáticos que aquellos con salud mental moderada, quienes a su vez tenían menores niveles de depresión que el grupo con salud mental languideciente. No se encontraron diferencias significativas en los sintomas somáticos entre el grupo de salud mental moderada y languideciente. Por otro lado, aquellas personas con salud mental floreciente mostraron también mayor satisfacción con la vida, mayor satisfacción en distintas áreas vitales y mayor presencia de significado en sus vidas en comparación con los individuos con salud mental moderada, quienes a su vez presentaban mayores niveles en estas variables que el grupo con salud mental languideciente. No se hallaron diferencias significativas entre los grupos para la Búsqueda de significado en la vida. Las diferencias más importantes se encontraron en los niveles de depresión, satisfacción en distintas áreas vitales y presencia de significado en la vida en las que el tamaño de efecto fue grande (Cohen, 1992). Las diferencias en la satisfacción con la vida resultaron de un tamaño de efecto moderado y bajas para los sintomas somáticos.

Tabla 1

Análisis de varianza de las variables de estudio de acuerdo con los niveles de salud mental.

\begin{tabular}{lccccc}
\hline Escala & $\begin{array}{c}\text { Salud Mental } \\
\text { Languideciente } \\
(n=25)\end{array}$ & $\begin{array}{c}\text { Salud Mental } \\
\text { Moderada } \\
(n=273)\end{array}$ & $\begin{array}{c}\text { Salud Mental } \\
\text { Floreciente } \\
(n=162)\end{array}$ & $\boldsymbol{F}$ & $\boldsymbol{\eta}^{2}$ \\
\cline { 2 - 5 } & $\boldsymbol{M}(\boldsymbol{D E})$ & $\boldsymbol{M}(\boldsymbol{D E})$ & $\boldsymbol{M}(\boldsymbol{D E})$ & & \\
\hline CES-D & $28.54(10.12) \mathrm{a}$ & $15.03(9.21) \mathrm{b}$ & $12.93(9.88) \mathrm{c}$ & $77.37^{* * *}$ & .27 \\
Shaffer & $18.68(8.45) \mathrm{a}$ & $19.06(8.64) \mathrm{a}$ & $15.75(6.13) \mathrm{b}$ & $7.90^{* * *}$ & .03 \\
SWLS & $19.36(7.21) \mathrm{a}$ & $23.69(5.57) \mathrm{b}$ & $27.32(5.12) \mathrm{c}$ & $34.70^{* * *}$ & .14 \\
PWI & $34.2(12.75) \mathrm{a}$ & $51.17(10.72) \mathrm{b}$ & $61.88(8.88) \mathrm{c}$ & $91.12^{* * *}$ & .30 \\
MLQ-P & $15.52(6.77) \mathrm{a}$ & $24.31(6.24) \mathrm{b}$ & $29.32(5.27) \mathrm{c}$ & $63.68^{* * *}$ & .23 \\
MLQ-B & $19.84(7.63)$ & $19.14(7.47)$ & $18.44(7.93)$ & $2.19 n s$ & .01 \\
\hline
\end{tabular}

***$p<.001$

Nota: SWLS = Satisfacción con la vida; PWI = Índice de Bienestar Personal; MLQ-P = Sentido de la vida- Presencia; $M L Q-S=$ Sentido de la vida- Búsqueda; $C E S-D=$ Depresión; Shaffer = Escala de síntomas somáticos. Las medias en la misma fila que no comparten los subíndices difieren a un nivel $p<.05$.

\section{Niveles de bienestar emocional, psicológico y social de acuerdo a las variables demográficas sexo, edad, ocupación y nivel educativo}

Se efectuó una prueba $t$ para comparar los niveles de bienestar emocional, psicológico, social y bienestar total entre hombres y mujeres evaluados a través del MHC-SF. El análisis no encontró diferencias significativas en ninguna de las variables dependientes según el sexo.

Posteriormente, se procedió a realizar diferentes análisis univariados de varianza (ANOVA) con análisis post-hoc de Tuckey para comparar los niveles de bienestar emocional, psicológico, social y total según grupo de edad, tipo de ocupación y nivel educativo máximo alcanzado. Este procedimiento post-hoc se seleccionó porque el análisis de homogeneidad de varianzas de Levene indicó que estas eran iguales entre los grupos.

En lo que se refiere al bienestar emocional, psicológico y total, no se encontraron diferencias significativas según grupo de edad -adultos jóvenes, adultos medios y adultos mayores- (tabla 2). Sin embargo, se encontró que en el bienestar social los adultos medios (30-59 años) presentaban mayores 
puntuaciones que los adultos mayores $\left(p=.02 ; \eta^{2}=.02\right)$. No obstante, la magnitud de esta diferencia es de un tamaño de efecto pequeño (Cohen, 1992).

Tabla 2

Análisis de varianza (ANOVA) del bienestar emocional, psicológico, social y total según grupo de edad.

\begin{tabular}{lcccc}
\hline \multirow{2}{*}{ Variable } & \multicolumn{3}{c}{ Edad } & \multirow{2}{*}{} \\
\cline { 2 - 4 } & $\begin{array}{c}\mathbf{1 8 - 2 9} \\
(n=127)\end{array}$ & $\begin{array}{c}\mathbf{3 0 - 5 9} \\
(n=281)\end{array}$ & $\begin{array}{c}\mathbf{6 0 +} \\
(n=52)\end{array}$ \\
\hline Bienestar Emocional $M(D E)$ & $10.53(2.93)$ & $10.86(4.03)$ & $10.78(3.63)$ & 0.34 \\
Bienestar Psicológico $M(D E)$ & $19.90(5.67)$ & $20.75(6.31)$ & $20.76(6.65)$ & 0.87 \\
Bienestar Social $M(D E)$ & $10.41(4.81)$ & $11.22(5.42)$ & $9.13(4.87)$ & $3.94^{*}$ \\
Bienestar Total $M(D E)$ & $42.67(12.14)$ & $40.52(11.79)$ & $43.32(12.36)$ & 1.49 \\
\hline
\end{tabular}

${ }^{*} p<.05$

Con respecto al nivel de educación máximo alcanzado (tabla 3), se encontraron diferencias significativas en los niveles de bienestar psicológico, social y total; en todos los casos fue mayor el nivel de bienestar en aquellas personas con un nivel educativo más alto. El análisis post-hoc reveló que en el bienestar psicológico las diferencias significativas se encontraban entre aquellos con educación de postgrado y educación primaria $\left(p<.05 ; \eta^{2}=.02\right), \mathrm{y}$ en el bienestar social entre aquellos con educación primaria y educación universitaria $\left(p<.05 ; \eta^{2}=.03\right)$. En cuanto al bienestar total $\left(\eta^{2}=.03\right)$, las diferencias se presentaron entre las personas con educación primaria y las personas con educación universitaria $(p<.05)$ y de postgrado $(p<.05)$.

Tabla 3

Análisis de varianza (ANOVA) del bienestar emocional, psicológico, social y total según nivel de educación máximo alcanzado

\begin{tabular}{|c|c|c|c|c|c|}
\hline \multirow[b]{2}{*}{ Variable } & \multicolumn{4}{|c|}{ Educación } & \multirow[b]{2}{*}{$\boldsymbol{F}$} \\
\hline & $\begin{array}{c}\text { Primario } \\
(n=41)\end{array}$ & $\begin{array}{c}\text { Secundario } \\
(n=222)\end{array}$ & $\begin{array}{l}\text { Univ/Terc } \\
(n=172)\end{array}$ & $\begin{array}{c}\text { Postgrado } \\
(n=25)\end{array}$ & \\
\hline Bienestar Emocional $M(D E)$ & $10.05(3.41)$ & $10.56(3.05)$ & $11.01(4.42)$ & $11.79(2.79)$ & 1.63 \\
\hline Bienestar Psicológico $M(D E)$ & $18.47(7.17)$ & $20.28(5.82)$ & $21.00(6.45)$ & $22.58(4.63)$ & $2.83^{*}$ \\
\hline Bienestar Social $M(D E)$ & $8.97(4.87)$ & 10.26(5.02) & 11.68(5.42) & $11.41(5.21)$ & $4.15^{\star *}$ \\
\hline Bienestar Total $M(D E)$ & $37.50(12.94)$ & 41.11(11.49) & $43.32(12.36)$ & $45.79(9.45)$ & $3.80^{* *}$ \\
\hline
\end{tabular}

Para analizar las diferencias en los distintos tipos de bienestar según tipo de ocupación (tabla 4), se decidió excluir al grupo de personas desempleadas $(n=6)$ dado que al ser tan pequeño podía afectar los resultados. El análisis mostró que la única diferencia estadísticamente significativa por tipo de ocupación fue en el bienestar social; los niveles de bienestar más bajos se presentaron en el grupo de ama de casas y personas que trabajan pero sin salario en comparación con el grupo de trabajadores independientes $(p<.05$; $\left.\eta^{2}=.03\right)$. Es importante destacar que en todos los casos, la magnitud de las diferencias significativas entre los grupos tiene un tamaño de efecto pequeño. 
Tabla 4

Análisis de varianza (ANOVA) del bienestar emocional, psicológico, social y total según tipo de ocupación

\begin{tabular}{|c|c|c|c|c|c|c|c|}
\hline \multirow[b]{2}{*}{ Variable } & \multicolumn{6}{|c|}{ Tipo de Ocupación } & \multirow[b]{2}{*}{$F$} \\
\hline & $\begin{array}{l}\text { Patrón } \\
(n=40)\end{array}$ & $\begin{array}{c}\text { Empleado } \\
(n=261)\end{array}$ & $\begin{array}{l}\text { Trab. Indep. } \\
\quad(n=75)\end{array}$ & $\begin{array}{c}\text { Ama de } \\
\text { casa/sin salario } \\
(n=17)\end{array}$ & $\begin{array}{c}\text { Jubilado } \\
(n=24)\end{array}$ & $\begin{array}{c}\text { No trabajo } \\
\quad(n=37)\end{array}$ & \\
\hline Bienestar Emocional $M(D E)$ & $10.17(3.84)$ & $10.88(3.83)$ & $11.25(2.83)$ & $8.88(4.31)$ & $10.56(3.47)$ & $11.44(5.07)$ & 1.64 \\
\hline Bienestar Psicológico $M(D E)$ & $20.07(5.83)$ & $20.61(6.34)$ & $20.92(5.13)$ & $16.94(7.85)$ & $20.87(7.17)$ & $21.18(4.97)$ & 1.16 \\
\hline Bienestar Social $M(D E)$ & $11.47(5.88)$ & $10.72(5.02)$ & $11.73(5.85)$ & $7.82(2.94)$ & $8.54(4.72)$ & $11.44(5.07)$ & $2.36^{*}$ \\
\hline Bienestar Total $M(D E)$ & $33.60(12.94)$ & $39.60(12.77)$ & $37.83(17.88)$ & $43.41(10.73)$ & $39.60(12.77)$ & $43.41(10.73)$ & 2.08 \\
\hline
\end{tabular}

${ }^{*} p<.05$

\section{DISCUSIÓN}

El primer objetivo de este estudio fue comparar los niveles de satisfacción con la vida, el significado en la vida, la satisfacción en distintas áreas vitales, el nivel de depresión y de síntomas somáticos entre adultos de la ciudad de Buenos Aires en las distintas categorias del continuo de salud mental. Los resultados mostraron que, conforme a las hipótesis, aquellos adultos que se encontraban en la categoria de salud mental floreciente presentaban mayores niveles en las variables positivas: satisfacción con la vida, satisfacción en distintas áreas vitales y presencia de significado en la vida en comparación con los adultos en la categoría siguiente salud mental moderada, quienes a su vez presentaban mayores niveles de estas variables que los adultos con salud mental languideciente. De igual forma, las variables patológicas - depresión y sintomas somáticos-resultaron menores en el grupo de salud mental floreciente. Estos hallazgos están en consonancia con los postulados de Keyes (2002) sobre el continuo de salud mental y con las investigaciones previas que sostienen que los adultos con alta salud mental (flourishing) funcionan en todos los aspectos mejor que los adultos con salud mental moderada, que a su vez funcionaban mejor que los adultos en la categoria languishing (Karaś et al., 2014; Keyes, 2007; Petrillo et al., 2015). El flourishing o florecimiento sería el estado óptimo de la salud mental y se asocia con menores niveles de patología como depresión, y síntomas somáticos (Keyes, 2005).

El segundo objetivo de este trabajo se refirió a comparar los niveles de bienestar emocional, psicológico y social en adultos de la ciudad de Buenos Aires según sexo, grupo de edad, ocupación y nivel educativo. Conforme a la hipótesis formulada, se encontraron mayores niveles de bienestar psicológico y social en adultos con mayor nivel educativo. Estos resultados son similares a la mayoría de los estudios internacionales y es el indicador demográfico con resultados más consistentes en la literatura (Karaś et al., 2014; Keyes, 2005; Lim et al., 2013; Yin et al., 2013). Se podría suponer que el bienestar emocional, al estar más vinculado a cuestiones biológicas (Lyubomirsky, 2007), está menos afectado por el nivel educativo. Existe amplia evidencia de que aproximadamente el $50 \%$ del nivel de emociones positivas de una persona se deben a componentes genéticos (Lyubomirsky, 2007). Esto implica que al estar condicionado biológicamente existe menos probabilidad de cambio. 
En forma similar a los estudios con adultos estadounidenses y canadienses, no se hallaron diferencias en los niveles de bienestar por tipo de empleo pero sí entre aquellos que no recibian salario (amas de casa y trabajadores sin salario) y los trabajadores independientes (Gilmour, 2014; Keyes, 2007). Asimismo, un estudio chino previo encontró también que los trabajadores independientes presentaron mayor salud mental (Yin et al., 2013). Debe señalarse que, al tener un grupo muy pequeño de personas desempleadas en la muestra $(\mathrm{n}=6)$, no pudieron incluirse en los análisis, lo que impidió comparar los niveles de bienestar entre personas empleadas y desempleadas y ampliar así el espectro de análisis. Futuros estudios deberán contar con una mayor representación de personas desempleadas para poder reevaluar los resultados hallados.

Por otro lado, en coincidencia con estudios canadienses, coreanos y chinos, no se encontraron diferencias en los distintos tipos de bienestar entre hombres y mujeres de la ciudad de Buenos Aires (Gilmour, 2014; Lim et al., 2013; Yin et al., 2013). En el caso del estudio coreano, si bien no se encontraron diferencias por sexo, sí se reportó mayor nivel de bienestar emocional en mujeres con un nivel alto de salario, es decir, cuando cruzaron la variable sexo con otra, en este caso, nivel de ingresos, se hallaron diferencias entre hombres y mujeres (Lim et al., 2013). Tal como se mencionó anteriormente, en la presente investigación las personas que no recibian salario, entre ellas amas de casa, tenían un menor nivel de bienestar. Sería importante que en futuros estudios con una muestra más amplia se pueda evaluar el nivel de bienestar teniendo en cuenta la interacción de sexo y ocupación.

Por último, en cuanto a los grupos de edades, los resultados indicaron que al igual que en el estudio italiano (Petrillo et al., 2015) los adultos de edad media (30-60 años) mostraban más bienestar social que los adultos mayores. Westerhof y Keyes (2010) encontraron que el bienestar psicológico disminuía con la edad pero el bienestar social permanecía constante con los años en adultos mayores holandeses. Se puede observar una amplia variabilidad en los resultados de los estudios internacionales sobre los grupos de edad y los niveles de bienestar que tienen relación con las caracteristicas sociales y culturales de los grupos. Podría pensarse que las condiciones sociales de los adultos mayores de la ciudad de Buenos Aires se asocien con una menor integración social en la comunidad, y de esta forma se puede dar cuenta de las diferencias encontradas.

Dentro de las limitaciones del estudio pueden mencionarse la conformación de algunos grupos de ocupación muy pequeños, el ya mencionado grupo de desempleados así como de jubilados y amas de casa. Por otro lado, el estudio ha tenido un carácter transversal, lo que no permite evaluar el valor predictivo y la evolución de los niveles de bienestar en adultos. Por último, la muestra elegida ha sido de tipo intencional, lo que limita la generalización de los resultados a otros grupos de adultos de otras regiones del país. Futuras investigaciones podrian incluir adultos de otras regiones del país.

Este estudio ha podido verificar la hipótesis que la población adulta de la ciudad de Buenos Aires con salud mental floreciente presenta mayores características positivas y menos sintomatología somática y depresiva que otros adultos con menores niveles de salud mental. Asimismo, los adultos con mayores niveles de educación, los de edad media y los trabajadores 
independientes son quienes reportan mayores niveles de bienestar. Estos resultados corroboran hallazgos internacionales y proveen evidencia en población argentina de la teoría del bienestar de Keyes (2005). Sería necesario avanzar en estudios longitudinales sobre la evolución de los niveles de salud mental en población argentina, su interacción con variables positivas $y$ factores de riesgo para comprender mejor su estabilidad y los factores que inciden en su aumento o disminución a lo largo del tiempo. También sería importante diseñar y evaluar intervenciones destinadas a promover el bienestar en la población basadas en este modelo. La promoción de la salud mental positiva resulta de relevancia no solo para mejorar el bienestar de las personas sino para estas puedan alcanzar el flourishing.

\section{REFERENCIAS}

Casas, F., Sarriera, J., Abs, D., Coenders, G., Alfaro, J., Saforcada, E., \& Tonon, G. (2012). Subjective Indicators of Personal Well-Being among Adolescents. Performance and Results for Different Scales in Latin-Language Speaking Countries: A Contribution to the International Debate. Child Indicators Research, 5(1), 1-28. https://doi.org/10.1007/s12187-011-9119-1

Castro Solano, A. \& Diaz Morales, J. (2000). Estilos de personalidad, objetivos de vida y satisfacción vital. En M. P. Sanchez Lopez \& M. M. Casullo (Eds.), Los estilos de personalidad. Una perspectiva Iberoamericana (pp. 195-231). Madrid: Miño y Dávila.

Casullo, M. M. (1998). Evaluación de sintomas depresivos en estudiantes adolescentes. En M. M. Casullo (Ed.), Adolescentes en riesgo: Identificación y orientación psicológica (pp. 29-68). Buenos Aires: Paidos.

Cohen, J. (1992). Statistical Power Analysis. Current Directions in Psychological Science, 1(3), 98-101. https://doi.org/10.1111/1467-8721.ep10768783

Cummins, R. A. (1998). The Second Approximation to an International Standard for Life Satisfaction. Social Indicators Research, 43(3), 307-334. https://doi.org/10.1023/A:1006831107052

Diener, E. (2009). Subjective well-being. En E. Diener (Ed.), The science of well-being: The collected works of Ed Diener (pp. 11-58). New York, NY: Springer Science + Business Media. https://doi.org/10.1007/978-90-481-2350-6_2

Diener, E., Emmons, R. A., Larsen, R. J., \& Griffin, S. (1985). The Satisfaction With Life Scale. Journal of Personality Assessment, 49(1), 71-75. https://doi.org/10.1207/s15327752jpa4901_13

Diener, E., Wirtz, D., Biswas-Diener, R., Tov, W., Kim-Prieto, C., Choi, D.-W., \& Oishi, S. (2009). New measures of well-being. En E. Diener (Ed.), Assessing well-being: The collected works of Ed Diener (pp. 247-266). New York, NY: Springer Science + Business Media. https://doi.org/10.1007/978-90-4812354-4_12

Diener, E., Wirtz, D., Tov, W., Kim-Prieto, C., Choi, D.-w., Oishi, S., \& Biswas-Diener, R. (2010). New Wellbeing Measures: Short Scales to Assess Flourishing and Positive and Negative Feelings. Social Indicators Research, 97(2), 143-156. doi: 10.1007/s11205-009-9493-y

Gallagher, M. W., Lopez, S. J., \& Preacher, K. J. (2009). The Hierarchical Structure of Well-Being. Journal of personality, 77(4), 1025-1050. https://doi.org/10.1111/j.1467-6494.2009.00573.x

Gilmour, H. (2014). Positive mental health and mental illness. Health reports, 25(9), 3-9.

Góngora, V. C. \& Castro Solano, A. (2011). Validación del Cuestionario de Significado de la Vida MLQ en población adulta y adolescente argentina. Revista Interamericana de Psicologí, 45(3), 395-404.

Góngora, V. C. \& Castro Solano, A. (2017). Assessment of the Mental Health Continuum in a sample of Argentinean Adults. Psychology, 8, 303-318. https://doi.org/10.4236/psych.2017.83018

Henderson, L. W. \& Knight, T. (2012). Integrating the hedonic and eudaimonic perspectives to more comprehensively understand wellbeing and pathways to wellbeing. International Journal of Wellbeing, 2(3), 196-221. https://doi.org/10.5502/ijw.v2i3.3

Hone, L. C., Jarden, A., Schofield, G. M., \& Duncan, S. (2014). Measuring flourishing: The impact of operational definitions on the prevalence of high levels of wellbeing. International Journal of Wellbeing, 4(1), 62-90. https://doi.org/10.5502/ijw.v4i1.1

Huppert, F. \& So, T. C. (2013). Flourishing Across Europe: Application of a New Conceptual Framework for Defining Well-Being. Social Indicators Research, 110(3), 837-861. https://doi.org/10.1007/s11205011-9966-7

Joshanloo, M., Wissing, M. P., Khumalo, I. P., \& Lamers, S. M. A. (2013). Measurement invariance of the Mental Health Continuum-Short Form (MHC-SF) across three cultural groups. Personality and Individual Differences, 55(7), 755-759. https://doi.org/10.1016/j.paid.2013.06.002

Karaś, D., Cieciuch, J., \& Keyes, C. L. M. (2014). The Polish adaptation of the Mental Health ContinuumShort Form (MHC-SF). Personality and Individual Differences, 69(0), 104-109. doi: https://doi.org/10.1016/j.paid.2014.05.011

Keyes, C. L. M. (2005). Mental illness and/or mental health? Investigating axioms of the complete state model of health. Journal of consulting and clinical psychology, 73(3), 539-548. https: / / doi.org/ 10.1037/0022-006X.73.3.539

Keyes, C. L. M. (2007). Promoting and protecting mental health as flourishing: a complementary strategy for 
improving national mental health. The American Psychologist, 62(2), 95-108. https://doi.org/10.1037/0003-066X.62.2.95

Keyes, C. L. M., Dhingra, S. S., \& Simoes, E. J. (2010). Change in Level of Positive Mental Health as a Predictor of Future Risk of Mental Illness. American Journal of Public Health, 100(12), 2366-2371. https://doi.org/10.2105/AJPH.2010.192245

Keyes, C. L. M., Eisenberg, D., Perry, G. S., Dube, S. R., Kroenke, K., \& Dhingra, S. S. (2011). The Relationship of Level of Positive Mental Health With Current Mental Disorders in Predicting Suicidal Behavior and Academic Impairment in College Students. Journal of American College Health, 60(2), 126-133. https://doi.org/10.1080/07448481.2011.608393

Keyes, C. L. M., \& Lopez, S. J. (2002). Toward a science of mental health: Positive directions in diagnosis and interventions. En S. J. Lopez \& C. R. Snyder (Eds.), Handbook of positive psychology (pp. 45-59). New York, NY: Oxford University Press.

Keyes, C. L. M. \& Simoes, E. J. (2012). To Flourish or Not: Positive Mental Health and All-Cause Mortality. American Journal of Public Health, 102(11), 2164-2172. https://doi.org/10.2105/AJPH.2012.300918

Lim, Y.-J., Ko, Y.-G., Shin, H.-C., \& Cho, Y. (2013). Prevalence and Correlates of Complete Mental Health in the South Korean Adult Population. En C. L. M. Keyes (Ed.), Mental Well-Being (pp. 91-109): Springer Netherlands. https://doi.org/10.1007/978-94-007-5195-8_5

Lupano Perugini, M. L., de la Iglesia, G., Castro Solano, A., \& Keyes, C. L. M. (2017). The Mental Health Continuum-Short Form (MHC-SF) in the Argentinean Context: Confirmatory Factor Analysis and Measurement Invariance. Europe's Journal of Psychology, 13(1), 93-108. https://doi.org/10.5964/ejop.v13i1.1163

Lyubomirsky, S. (2007). The how of happiness: A scientific approach to getting the life you want. New York, NY, US: Penguin Press.

Lyubomirsky, S. \& Lepper, H. (1999). A Measure of Subjective Happiness: Preliminary Reliability and Construct Validation. Social Indicators Research, 46(2), 137-155. https://doi.org/10.1023/A:1006824100041

Pavot, W. \& Diener, E. (2008). The Satisfaction With Life Scale and the emerging construct of life

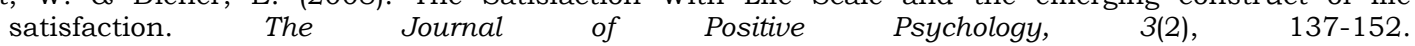
https://doi.org/10.1080/17439760701756946

Pavot, W., Diener, E., Colvin, C. R., \& Sandvik, E. (1991). Further validation of the Satisfaction With Life Scale: Evidence for the cross-method convergence of well-being measures. Journal of Personality Assessment, 57(1), 149-161. https://doi.org/10.1207/s15327752jpa5701_17

Petrillo, G., Capone, V., Caso, D., \& Keyes, C. L. M. (2015). The Mental Health Continuum-Short Form (MHC-SF) as a Measure of Well-Being in the Italian Context. Social Indicators Research, 121(1), 291312. https://doi.org/10.1007/s11205-014-0629-3

Radloff, L. S. (1977). The CES-D Scale: A Self-Report Depression Scale for Research in the General $\begin{array}{llll}\text { Population. } & \text { Applied } \quad \text { Psychological } & \text { Measurement, } & 1(3),\end{array}$ https://doi.org/10.1177/014662167700100306

Ryan, R. M. \& Deci, E. L. (2001). On happiness and human potentials: A Review of Research on Hedonic and Eudaimonic Well-Being. Annual Review of Psychology, 52(1), 141-166. https://doi.org/10.1146/annurev.psych.52.1.141

Ryff, C. D. (1989). Happiness is everything, or is it? Explorations on the meaning of psychological wellbeing. Journal of Personality and Social Psychology, 57(6), 1069-1081. https://doi.org/10.1037/00223514.57.6.1069

Seligman, M. E. P. (2011). Flourish: A visionary new understanding of happiness and well-being. New York: Free Press.

Schafer, M. H. \& Ferraro, K. F. (2013). Childhood Misfortune and Adult Health: Enduring and Cascadic Effects on Somatic and Psychological Symptoms? Journal of Aging and Health, 25(1), 3-28. https://doi.org/10.1177/0898264312464884

Steger, M. F., Frazier, P., Oishi, S., \& Kaler, M. (2006). The Meaning in Life Questionnaire: Assessing the Presence of and Search for Meaning in Life. Journal of Counseling Psychology, 53(1), 80-93. https://doi.org/10.1037/0022-0167.53.1.80

Tonon, G. (2012). Quality of Life in Argentina. En K. C. Land, A. C. Michalos \& M. J. Sirgy (Eds.), Handbook of Social Indicators and Quality of Life Research (pp. 547-554): Springer Netherlands. https://doi.org/10.1007/978-94-007-2421-1_25

Westerhof, G. \& Keyes, C. L. M. (2010). Mental Illness and Mental Health: The Two Continua Model Across the Lifespan. Journal of Adult Development, 17(2), 110-119. https://doi.org/10.1007/s10804-0099082-y

Yin, K., He, J., \& Fu, Y. (2013). Positive Mental Health: Measurement, Prevalence, and Correlates in a Chinese Cultural Context. En C. L. M. Keyes (Ed.), Mental Well-Being (pp. 111-132): Springer Netherlands. https://doi.org/10.1002/pmh.1216

Recibido 13-11-2017 | Aceptado 16-03-2018

Este trabajo se encuentra bajo una Licencia Creative Commons Atribución 4.0 Internacional que permite a terceros utilizar lo publicado siempre que se dé el crédito pertinente a los autores y a Psicodebate 